\title{
ON POSITIVE SOLUTIONS FOR A SECOND ORDER ORDINARY DIFFERENTIAL SYSTEM
}

\author{
XU YANG ${ }^{1}$, YUANFANG RU $^{2, *}$, FANGLEI WANG $^{3}$ \\ ${ }^{1}$ Depatment of Mathematics, College of Science, Hohai University, Nanjing 210098, China \\ ${ }^{2}$ College of Science, China pharmaceutical University, Nanjing 211198, China
}

Abstract. By using the fixed point index theory, we study the existence and multiplicity of positive solutions for a second order ordinary differential system.

Keywords. Ordinary differential equation; Positive solution; Cone; Fixed point theorem; Fixed point index.

2010 Mathematics Subject Classification. 34B18.

\section{INTRODUCTION}

This paper deals with the existence of positive solutions of nonlinear differential system

$$
\begin{cases}-u^{\prime \prime}+b u=\phi u+f(t, u, \phi), & 0<t<1 \\ -\phi^{\prime \prime}=\mu u, & 0<t<1 \\ u(0)=u(1)=\phi(0)=\phi(1)=0, & \end{cases}
$$

where $b, \mu>0$ and $f:[0,1] \times R^{+} \times R^{+} \rightarrow R^{+}$is continuous.

During the last few decades, the second order elliptic systems

$$
\begin{cases}-\Delta u+b u=\phi u+f(u), & x \in \Omega, \\ -\Delta \phi=\mu u, & x \in \Omega, \\ u=\phi=0, \& x \in \partial \Omega & \end{cases}
$$

have been widely investigated because they have a lot of applications in quantum mechanics models $[1,2]$, semiconductor theory [3] or a time and space-dependent mathematical model of nuclear reactors

${ }^{*}$ Corresponding author.

E-mail addresses: yangxu940910@163.com (X. Yang), ruyanfangmm@163.com (Y. Ru), wang-fanglei@ hotmail.com (F. Wang).

Received March 15, 2019; Accepted June 27, 2019. 
in a closed container [4]. Recently, Gu and Wang [5] proved the existence of positive solutions of the following elliptic system

$$
\begin{cases}-\Delta u+b u=\phi u, & x \in \Omega, \\ -\Delta \phi=u, & x \in \Omega, \\ u=\phi=0, & x \in \partial \Omega\end{cases}
$$

by using the abstract fixed point theorem. In addition, they also proved that every positive stationary solution of (1.2) is a threshold when $\Omega \in R^{N}(2 \leq N<6)$ is a ball. In 2011, Wang and An [6] investigated the existence of positive solutions of the following one-dimensional system

$$
\begin{cases}-u^{\prime \prime}+b u=\phi u+f(t, u), & 0<t<1, \\ -\phi^{\prime \prime}=\mu u, & 0<t<1 \\ u(0)=u(1)=\phi(0)=\phi(1)=0 . & \end{cases}
$$

By using Krasnosel'skii's fixed point theorem, they provided sufficient conditions for the existence of at least one positive solution to the BVP (1.3). In 2012, Chen and Ma [7] applied the bifurcation techniques to study the existence of solutions for (1.3). They also established some existence results for the BVP

$$
\begin{cases}-\Delta u+b u=\phi u+f(|x|, u), & R_{1}<|x|<R_{2}, x \in R^{N}, N \geq 1 \\ -\Delta \phi=\mu u, & R_{1}<|x|<R_{2}, x \in R^{N}, N \geq 1 \\ u=\phi=0, & \text { on }|x|=R_{1},|x|=R_{2}\end{cases}
$$

by using bifurcation techniques. Recently, Wang and An [8] considered the existence of positive solutions for a second order differential system

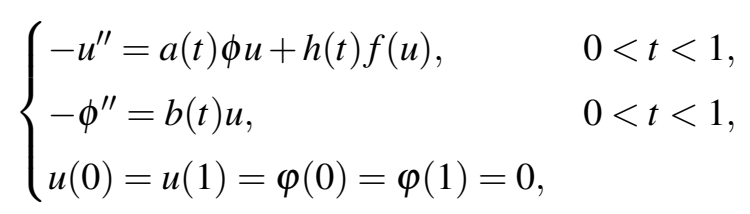

where $a(t), b(t), h(t)$ may change sign. By using the Krasnosel'skii's fixed point theorem, they obtained sufficient conditions that guarantee the existence of at least one positive solution.

In above results, one of the common techniques is the Green function, and the solution of the boundary value problem

$$
-\phi^{\prime \prime}=\mu u, \phi(0)=\phi(1)=0
$$

can be expressed as

$$
\phi(t)=\mu \int_{0}^{1} G(t, s) u(s) d s .
$$

Then, inserting (1.4) into the first equation of (1.3), we get a second-order nonlocal boundary vale problem

$$
\left\{\begin{array}{l}
-u^{\prime \prime}+\lambda u=\mu u \int_{0}^{1} G(t, s) u(s) d s+f(t, u), 0<t<1 \\
u(0)=u(1)=0
\end{array}\right.
$$


But, if we insert the second equation (1.1) into the first equation of (1.1), then we can obtain a fourthorder boundary value problem

$$
\left\{\begin{array}{l}
\phi^{(4)}-b \phi^{\prime \prime}=-\phi \phi^{\prime \prime}+\mu f\left(t, \phi, \frac{-\phi^{\prime \prime}}{\mu}\right), \quad 0<t<1, \\
\phi(0)=\phi(1)=0, \phi^{\prime \prime}(0)=\phi^{\prime \prime}(1)=0 .
\end{array}\right.
$$

To the best of our knowledge, the existence and multiplicity of nontrivial solutions for the fourth-order differential equation

$$
u^{(4)}=f\left(t, u, u^{\prime}, u^{\prime \prime}, u^{\prime \prime \prime}\right)
$$

with various boundary conditions have been widely studied by using variational methods, the method of upper and lower solutions, fixed point theorems, the alternative principle of Leray-Schauder or the topological degree theory. We refer the readers to $[9,10,11,12,13,14,15,16,17,18,19,20,21,22,23$, $24]$ and the references therein. For fourth-order BVP (1.5), there are few literatures on the existence of positive solutions. In this paper, we apply the fixed point index theory in a cone to discuss the existence and multiplicity of positive solutions of the fourth-order BVP (1.5). Compared to the results in the previous works, our results presented in this paper has the following new features. First, we find some new conditions, which differ from those in the majority of results as we know. Second, the results we are going to present reveal how the behavior of the functions $\phi \phi^{\prime \prime}$ and $f$ have a profound effect on the existence and multiplicity of positive solutions of (1.5) under some appropriate conditions.

\section{PRELIMINARIES}

Let $G(t, s)$ be the Green function of linear boundary value problem

$$
-u^{\prime \prime}+\lambda u=0, u(0)=u(1)=0
$$

where $\lambda>-\pi^{2}$.

Lemma 2.1. [15] Let $\omega=\sqrt{|\lambda|}$. Then $G(t, s)$ can be expressed by

(i) $G(t, s)=\left\{\begin{array}{ll}\frac{\sinh \omega t \sinh \omega(1-s)}{\omega \sinh \omega}, & 0 \leq t \leq s \leq 1, \\ \frac{\sinh \omega \sinh \omega(1-t)}{\omega \sinh \omega}, & 0 \leq s \leq t \leq 1,\end{array}\right.$ if $\lambda>0$,

(ii) $G(t, s)=\left\{\begin{array}{ll}t(1-s), & 0 \leq t \leq s \leq 1, \\ s(1-t), & 0 \leq s \leq t \leq 1,\end{array}\right.$ if $\lambda=0$,

(iii) $G(t, s)=\left\{\begin{array}{ll}\frac{\sin \omega t \sin \omega(1-s)}{\omega \sin \omega}, & 0 \leq t \leq s \leq 1, \\ \frac{\sin \omega \sin \omega(1-t)}{\omega \sin \omega}, & 0 \leq s \leq t \leq 1,\end{array}\right.$ if $-\pi^{2}<\lambda<0$.

Lemma 2.2. [15] The function $G(t, s)$ has the following properties:

(i) $G(t, s)>0, \forall t, s \in(0,1)$,

(ii) $G(t, s) \leq C G(s, s), \forall t, s \in[0,1]$,

(iii) $G(t, s) \geq \delta G(t, t) G(s, s), \forall t, s \in[0,1]$,

where $C=1, \delta=\omega / \sinh \omega$, if $\lambda>0 ; C=1, \delta=1$, if $\lambda=0 ; C=1 / \sin \omega, \delta=\omega \sin \omega$, if $-\pi^{2}<\lambda<0$. 
Let $G(t, s), G_{b}(t, s)$ denote the Green function for $\lambda=0$ and $\lambda=b$, respectively. Now the solutions of (1.5) can be rewritten as fixed points of operators $\mathfrak{T}$ in an appropriate Banach space, where

$$
\begin{aligned}
\mathfrak{T} \phi(t)= & -\int_{0}^{1} \int_{0}^{1} G(t, s) G_{b}(s, \tau) \phi(\tau) \phi^{\prime \prime}(\tau) d \tau d s \\
& +\mu \int_{0}^{1} \int_{0}^{1} G(t, s) G_{b}(s, \tau) f\left(\tau, \phi(\tau), \frac{-\phi^{\prime \prime}(\tau)}{\mu}\right) d \tau d s .
\end{aligned}
$$

Let $E$ denote the the Banach space $C^{2}[0,1]$ with the norm

$$
\|u\|=|u|_{\infty}+\left|u^{\prime \prime}\right|_{\infty}=\max _{t \in[0,1]}|u(t)|+\max _{t \in[0,1]}\left|u^{\prime \prime}(t)\right|, \text { for } u \in C^{2}[0,1] .
$$

Define a cone $K \subset E$ by

$$
K=\left\{u(t) \in C^{2}[0,1]: u \geq 0, u^{\prime \prime} \leq 0, \min _{t \in\left[\frac{1}{4}, \frac{3}{4}\right]} u(t) \geq \sigma_{1}|u|_{\infty}, \max _{t \in\left[\frac{1}{4}, \frac{3}{4}\right]} u^{\prime \prime}(t) \leq-\sigma_{2}\left|u^{\prime \prime}\right|_{\infty}\right\},
$$

where $\sigma_{i} \in(0,1)$ (see [15]). Also, for $r>0$, define $K_{r}$ and $\partial K_{r}$ by

$$
K_{r}=\{u(t) \in K:\|u\|<r\}, \quad \partial K_{r}=\{u(t) \in K:\|u\|=r\} .
$$

Lemma 2.3. Let $h \in C^{+}[0,1]$. Then the linear boundary value problem

$$
\left\{\begin{array}{l}
u^{(4)}-b u^{\prime \prime}=h(t), 0<t<1, \\
u(0)=u(1)=0, u^{\prime \prime}(0)=u^{\prime \prime}(1)=0
\end{array}\right.
$$

has a unique solution $u(t)$, which satisfies the following estimates:

(i) $u(t) \geq G(t, t)|u|_{\infty}$, for every $t \in[0,1]$, and $|u|_{\infty} \leq \frac{\pi^{3}}{4} \int_{0}^{1} u(t) \sin \pi t d s$.

(ii) $u^{\prime \prime}(t) \leq-\delta_{b} G_{b}(t, t)\left|u^{\prime \prime}\right|_{\infty}$, for every $t \in[0,1]$, and

$$
\left|u^{\prime \prime}\right|_{\infty} \leq \Gamma \cdot \int_{0}^{1} u(t) \sin \pi t d s
$$

where

$$
\Gamma=\left(\pi^{3}+\frac{\pi^{5}}{4 b^{2}}\right) \cdot \sinh \sqrt{b} \cdot \tanh \sqrt{b}
$$

Proof. Let $v=-u^{\prime \prime}$. Then,

$$
v(t)=\int_{0}^{1} G_{b}(t, s) h(s) d s
$$

and

$$
u(t)=\int_{0}^{1} G(t, s) v(s) d s
$$

From Lemma 2.2, it follows that

$$
\begin{aligned}
u(t) & =\int_{0}^{1} G(t, s) v(s) d s \\
& \geq \int_{0}^{1} G(t, t) G(s, s) v(s) d s \\
& \geq t(1-t)|u|_{\infty} .
\end{aligned}
$$

Multiplying both sides of the above inequality by $\sin \pi t$ and integrating on $[0,1]$, we get

$$
\int_{0}^{1} u(t) \sin \pi t d t \geq \int_{0}^{1} t(1-t) \sin \pi t d t \cdot|u|_{\infty}
$$


namely,

$$
|u|_{\infty} \leq \frac{\pi^{3}}{4} \int_{0}^{1} u(t) \sin \pi t d t .
$$

So, (i) of Lemma 2.3 holds. As the similar argument, we also have

$$
v(t) \geq \delta_{b} G_{b}(t, t)|v|_{\infty} \Leftrightarrow u^{\prime \prime}(t) \leq-\delta_{b} G_{b}(t, t)\left|u^{\prime \prime}\right|_{\infty}, \forall t \in[0,1]
$$

and

$$
|v|_{\infty} \leq \frac{1}{\int_{0}^{1} \delta_{b} G_{b}(t, t) \sin \pi t d t} \int_{0}^{1} v(t) \sin \pi t d t
$$

Letting $\omega=\sqrt{b}$, we find

$$
\begin{aligned}
\int_{0}^{1} \delta_{b} G_{b}(t, t) \sin \pi t d t= & \int_{0}^{1} \frac{\omega}{\sinh \omega} \frac{\sinh \omega t \cdot \sinh \omega(1-t))}{\omega_{1} \sinh \omega} \sin \pi t d t \\
= & \frac{1}{(\sinh \omega)^{2}} \int_{0}^{1} \sinh \omega t \cdot \sinh \omega(1-t) \sin \pi t d t \\
= & \frac{1}{4(\sinh \omega)^{2}} \frac{-1}{\pi} \int_{0}^{1} e^{\omega}-e^{\omega-2 \omega t}-e^{2 \omega t-\omega}+e^{-\omega} d \cos \pi t \\
= & \frac{1}{4(\sinh \omega)^{2}} \frac{-1}{\pi}\left[-2\left(e^{\omega}+e^{-\omega_{1}}\right)-\int_{0}^{1} e^{\omega-2 \omega t}+e^{2 \omega t-\omega} d \cos \pi t\right] \\
= & \frac{1}{4(\sinh \omega)^{2}} \frac{-1}{\pi}\left[-2\left(e^{\omega}+e^{-\omega}\right)-\left(-2\left(e^{\omega}+e^{-\omega}\right)\right)\right. \\
& \left.+\int_{0}^{1} \cos \pi t(-2 \omega) e^{\omega_{1}-2 \omega t}+(2 \omega) e^{2 \omega t-\omega} d t\right] \\
= & \frac{1}{4(\sinh \omega)^{2}} \frac{-1}{\pi} \int_{0}^{1} \cos \pi t\left[(-2 \omega) e^{\omega-2 \omega t}+(2 \omega) e^{2 \omega t-\omega}\right] d t .
\end{aligned}
$$

Let

$$
\Lambda(t)=\int_{0}^{1} \cos \pi t(-2 \omega) e^{\omega-2 \omega t}+(2 \omega) e^{2 \omega t-\omega} d t
$$

Then,

$$
\begin{aligned}
\Lambda(t) & =\frac{1}{\pi} \int_{0}^{1}(-2 \omega) e^{\omega-2 \omega t}+(2 \omega) e^{2 \omega t-\omega} d(\sin \pi t) \\
& =\frac{1}{\pi}\left[0-\int_{0}^{1} \sin \pi t d\left[(-2 \omega) e^{\omega-2 \omega t}+(2 \omega) e^{2 \omega t-\omega}\right]\right. \\
& =\frac{2 \omega}{\pi} \int_{0}^{1} \sin \pi t\left[(-2 \omega) e^{\omega-2 \omega t}-(2 \omega) e^{2 \omega t-\omega}\right] d t \\
& =\frac{2 \omega}{\pi} \frac{-1}{\pi} \int_{0}^{1}(-2 \omega) e^{\omega-2 \omega t}-(2 \omega) e^{2 \omega t-\omega} d(\cos \pi t) \\
& =\frac{4 \omega^{4}}{\pi^{2}}\left[-2\left(e^{\omega}+e^{-\omega}\right)-\int_{0}^{1} \cos \pi t\left[(-2 \omega) e^{\omega-2 \omega t}+(2 \omega) e^{2 \omega t-\omega}\right] d t\right. \\
& =\frac{4 \omega^{4}}{\pi^{2}}\left[-2\left(e^{\omega}+e^{-\omega}\right)-\Lambda(t)\right] \\
& =\frac{4 b^{2}}{\pi^{2}}\left[-2\left(e^{\sqrt{b}}+e^{-\sqrt{b}}\right)-\Lambda(t)\right],
\end{aligned}
$$


which yields

$$
\Lambda(t)=\frac{-8 b^{2}\left(e^{\sqrt{b}}+e^{-\sqrt{b}}\right)}{\pi^{2}+4 b^{2}}
$$

It is easy to see that

$$
\left.\int_{0}^{1} \delta_{b} G_{b}(t, t)\right) \sin \pi t d t=\frac{2 b^{2}\left(e^{\sqrt{b}}+e^{-\sqrt{b}}\right)}{(\sinh \sqrt{b})^{2} \cdot \pi \cdot\left(\pi^{2}+4 b^{2}\right)}
$$

Furthermore,

$$
\begin{aligned}
\left\|u^{\prime \prime}\right\| & \leq \frac{1}{\left.\int_{0}^{1} \delta_{b} G_{b}(t, t)\right) \sin \pi t d s} \int_{0}^{1}\left(-u^{\prime \prime}(t)\right) \sin \pi t d t \\
& =\frac{(\sinh \sqrt{b})^{2} \cdot \pi^{3} \cdot\left(\pi^{2}+4 b^{2}\right)}{2 b^{2}\left(e^{\sqrt{b}}+e^{-\sqrt{b}}\right)} \int_{0}^{1} u(t) \sin \pi t d s \\
& =\sinh \sqrt{b} \tanh \sqrt{b} \cdot\left(\pi^{3}+\frac{\pi^{5}}{4 b^{2}}\right) \int_{0}^{1} u(t) \sin \pi t d s,
\end{aligned}
$$

which implies that (ii) of Lemma 2.3 holds.

Lemma 2.4. If $f:[0,1] \times R^{+} \times R^{+} \rightarrow R^{+}$is continuous, then $\mathfrak{T}(K) \subseteq K$ and $\mathfrak{T}: K \rightarrow K$ is completely continuous.

Proof. For any $\phi(t) \in K$, we have

$$
\begin{aligned}
\mathfrak{T} \phi(t)= & -\int_{0}^{1} \int_{0}^{1} G(t, s) G_{b}(s, \tau) \phi(\tau) \phi^{\prime \prime}(\tau) d \tau d s \\
& +\mu \int_{0}^{1} \int_{0}^{1} G(t, s) G_{b}(s, \tau) f\left(\tau, \phi(\tau), \frac{-\phi^{\prime \prime}(\tau)}{\mu}\right) d \tau d s \geq 0
\end{aligned}
$$

and

$$
\begin{aligned}
\mathfrak{T}^{\prime \prime} \phi(t)= & \int_{0}^{1} G_{b}(t, \tau) \phi(\tau) \phi^{\prime \prime}(\tau) d \tau \\
& -\mu \int_{0}^{1} G_{b}(t, \tau) f\left(\tau, \phi(\tau), \frac{-\phi^{\prime \prime}(\tau)}{\mu}\right) d \tau \leq 0 .
\end{aligned}
$$

Moreover,

$$
\begin{aligned}
\min _{t \in\left[\frac{1}{4}, \frac{3}{4}\right]} \mathfrak{T} \phi(t)= & \min _{t \in\left[\frac{1}{4}, \frac{3}{4}\right]}-\int_{0}^{1} \int_{0}^{1} G(t, s) G_{b}(s, \tau) \phi(\tau) \phi^{\prime \prime}(\tau) d \tau d s \\
& +\min _{t \in\left[\frac{1}{4}, \frac{3}{4}\right]} \mu \int_{0}^{1} \int_{0}^{1} G(t, s) G_{b}(s, \tau) f\left(\tau, \phi(\tau), \frac{-\phi^{\prime \prime}(\tau)}{\mu}\right) d \tau d s \\
\geq & \min _{t \in\left[\frac{1}{4}, \frac{3}{4}\right]}-\int_{0}^{1} \int_{0}^{1} G(t, t) G(s, s) G_{b}(s, \tau) \phi(\tau) \phi^{\prime \prime}(\tau) d \tau d s \\
& +\min _{t \in\left[\frac{1}{4}, \frac{3}{4}\right]} \mu \int_{0}^{1} \int_{0}^{1} G(t, t) G(s, s) G_{b}(s, \tau) f\left(\tau, \phi(\tau), \frac{-\phi^{\prime \prime}(\tau)}{\mu}\right) d \tau d s \\
= & \min _{t \in\left[\frac{1}{4}, \frac{3}{4}\right]} G(t, t)\left[-\int_{0}^{1} \int_{0}^{1} G(s, s) G_{b}(s, \tau) \phi(\tau) \phi^{\prime \prime}(\tau) d \tau d s\right] \\
& +\min _{t \in\left[\frac{1}{4}, \frac{3}{4}\right]} G(t, t)\left[\mu \int_{0}^{1} \int_{0}^{1} G(s, s) G_{b}(s, \tau) f\left(\tau, \phi(\tau), \frac{-\phi^{\prime \prime}(\tau)}{\mu}\right) d \tau d s\right] \\
= & \sigma_{1}|\mathfrak{T} \phi|_{\infty}
\end{aligned}
$$


and

$$
\begin{aligned}
\max _{t \in\left[\frac{1}{4}, \frac{3}{4}\right]} \mathfrak{T}^{\prime \prime} \phi(t)= & \max _{t \in\left[\frac{1}{4}, \frac{3}{4}\right]}\left[\int_{0}^{1} G_{b}(t, \tau) \phi(\tau) \phi^{\prime \prime}(\tau) d \tau-\mu \int_{0}^{1} G_{b}(t, \tau) f\left(\tau, \phi(\tau), \frac{-\phi^{\prime \prime}(\tau)}{\mu}\right) d \tau\right] \\
= & -\min _{t \in\left[\frac{1}{4}, \frac{3}{4}\right]}\left[-\int_{0}^{1} G_{b}(t, \tau) \phi(\tau) \phi^{\prime \prime}(\tau) d \tau+\mu \int_{0}^{1} G_{b}(t, \tau) f\left(\tau, \phi(\tau), \frac{-\phi^{\prime \prime}(\tau)}{\mu}\right) d \tau\right] \\
\leq & -\min _{t \in\left[\frac{1}{4}, \frac{3}{4}\right]}\left[-\int_{0}^{1} G_{b}(t, t) G_{b}(\tau, \tau) \phi(\tau) \phi^{\prime \prime}(\tau) d \tau\right. \\
& \left.+\mu \int_{0}^{1} G_{b}(t, t) G_{b}(\tau, \tau) f\left(\tau, \phi(\tau), \frac{-\phi^{\prime \prime}(\tau)}{\mu}\right) d \tau\right] \\
\leq & -\min _{t \in\left[\frac{1}{4}, \frac{3}{4}\right]} G_{b}(t, t)\left[-\int_{0}^{1} G_{b}(\tau, \tau) \phi(\tau) \phi^{\prime \prime}(\tau) d \tau\right. \\
& \left.+\mu \int_{0}^{1} G_{b}(\tau, \tau) f\left(\tau, \phi(\tau), \frac{-\phi^{\prime \prime}(\tau)}{\mu}\right) d \tau\right] \\
= & \sigma_{2}\left|\mathfrak{T}^{\prime \prime} \phi\right|_{\infty} .
\end{aligned}
$$

So, $\mathfrak{T}(K) \subseteq K$. Finally, it is standard to verify that $\mathfrak{T}$ is completely continuous, see, e.g., [25]. This completes the proof.

At the end of this section, we give the following two crucial lemmas.

Lemma 2.5. [25] Let $\mathfrak{T}: K \rightarrow K$ be a completely continuous mapping. If $\lambda \mathfrak{T} u \neq u$ for every $u \in \partial K_{r}$ and $0<\lambda \leq 1$, then $i\left(\mathfrak{T}, K_{r}, K\right)=1$.

Lemma 2.6. [25] Let $\mathfrak{T}: K \rightarrow K$ be a completely continuous mapping. If there exists an $e \in K \backslash \theta$ such that $u-\mathfrak{T} u \neq \tau$ e for every $u \in \partial K_{r}$, then $i\left(\mathfrak{T}, K_{r}, K\right)=0$.

\section{MAin ReSUltS}

Theorem 3.1. Assume that $f:[0,1] \times R^{+} \times R^{+} \rightarrow R^{+}$is continuous and satisfies the following assumptions:

$\left(F_{1}\right)$ There exist $\alpha_{1}>0, \beta_{1} \geq 0, r_{1}>0,0<\frac{\mu \alpha_{1}}{\pi^{4}}+\frac{\beta_{1}-b}{\pi^{2}}+\frac{\min \left\{1, \mu^{2}\right\}}{2 \pi^{4}} r_{1}<1$, such that

$$
f(t, \phi, u) \leq \alpha_{1} \phi+\beta_{1} u, \forall t \in[0,1], \phi \in\left[0, r_{1}\right], u \in\left[0, \mu r_{1}\right] .
$$

$\left(F_{2}\right)$ There exist $\alpha_{2} \geq 0, \beta_{2} \geq 0, \frac{\alpha_{2}}{2 \pi^{4}}+\frac{\beta_{2}-b}{\pi^{2}}>1$, and $h(t) \in C^{+}[0,1]$, such that

$$
f(t, \phi, u) \geq \alpha_{2} \phi+\beta_{2} u-h(t), \forall t \in[0,1], \phi \geq 0, u \geq 0 .
$$

Then (1.5) has at least one positive solution.

Proof. Set $r=\frac{\min \left\{1, \mu^{2}\right\}}{2} r_{1}$. We prove that $\lambda \mathfrak{T} \phi \neq \phi$ for $\phi \in \partial K_{r}$ and $0<\lambda \leq 1$. On the contrary, there exist $\bar{\phi} \in \partial K_{r}$ and $0<\bar{\lambda} \leq 1$ such that $\bar{\lambda} \mathfrak{T} \bar{\phi}=\bar{\phi}$, namely,

$$
\bar{\phi}^{(4)}(t)-b \bar{\phi}^{\prime \prime}(t)=-\bar{\lambda} \bar{\phi}(t) \bar{\phi}^{\prime \prime}(t)+\bar{\lambda} \mu f\left(t, \bar{\phi}(t),-\frac{1}{\mu} \bar{\phi}^{\prime \prime}(t)\right), 0 \leq t \leq 1
$$

and

$$
\bar{\phi}(0)=\bar{\phi}(1)=\bar{\phi}^{\prime \prime}(0)=\bar{\phi}^{\prime \prime}(1)=0 \text {. }
$$


For $\bar{\phi} \in \partial K_{r}$, we have

$$
|\bar{\phi}|_{\infty} \leq \frac{r_{1}}{2}, \quad\left|\frac{1}{\mu} \bar{\phi}^{\prime \prime}\right|_{\infty} \leq \frac{\frac{\min \left\{1, \mu^{2}\right\}}{2}}{\mu} r_{1} \leq \frac{\frac{\mu^{2}}{2}}{\mu} r_{1}<\mu r_{1} .
$$

From $\left(F_{1}\right)$, it follows that

$$
\begin{aligned}
\bar{\phi}^{(4)}(t)-b \bar{\phi}^{\prime \prime}(t) & =-\bar{\lambda} \bar{\phi}(t) \bar{\phi}^{\prime \prime}(t)+\bar{\lambda} \mu f\left(t, \bar{\phi}(t),-\frac{1}{\mu} \bar{\phi}^{\prime \prime}(t)\right) \\
& \leq-\bar{\lambda} \bar{\phi}(t) \bar{\phi}^{\prime \prime}(t)+\bar{\lambda} \mu\left[\alpha_{1} \bar{\phi}(t)-\beta_{1} \frac{1}{\mu} \bar{\phi}^{\prime \prime}(t)\right] \\
& \left.=-\bar{\lambda} \bar{\phi}(t) \bar{\phi}^{\prime \prime}(t)+\bar{\lambda} \mu \alpha_{1} \bar{\phi}(t)-\bar{\lambda} \beta_{1} \bar{\phi}^{\prime \prime}(t)\right] \\
& \leq-\bar{\phi}(t) \bar{\phi}^{\prime \prime}(t)+\mu \alpha_{1} \bar{\phi}(t)-\beta_{1} \bar{\phi}^{\prime \prime}(t) \\
& \leq \bar{\phi}(t) \frac{\min \left\{1, \mu^{2}\right\}}{2} r_{1}+\mu \alpha_{1} \bar{\phi}(t)-\beta_{1} \bar{\phi}^{\prime \prime}(t) .
\end{aligned}
$$

Furthermore, multiplying this inequality by $\sin \pi t$ and integrating on $[0,1]$, we have

$$
\int_{0}^{1} \bar{\phi}(t) \sin \pi t d t \leq \frac{\left[\mu \alpha_{1}+\frac{\min \left\{1, \mu^{2}\right\}}{2} r_{1}+\left(\beta_{1}-b\right) \pi^{2}\right]}{\pi^{4}} \int_{0}^{1} \bar{\phi}(t) \sin \pi t d t .
$$

By Lemma 2.3, we have

$$
\|\bar{\phi}\|=|\phi|_{\infty}+\left|\bar{\phi}^{\prime \prime}\right|_{\infty} \leq\left(\Gamma+\frac{\pi^{3}}{4}\right) \int_{0}^{1} \bar{\phi}(t) \sin \pi t d t,
$$

which implies $\int_{0}^{1} \bar{\phi}(t) \sin \pi t d t>0$. Using (3.2), we get

$$
1 \leq \frac{\left[\mu \alpha_{1}+\frac{\min \left\{1, \mu^{2}\right\}}{2} r_{1}+\left(\beta_{1}-b\right) \pi^{2}\right]}{\pi^{4}}<1,
$$

which is a contradiction. Thus, $i\left(\mathfrak{T}, K_{r}, K\right)=1$.

Now, we choose a sufficiently large $R>\max \{r, \mu r\}$ and $e=\sin \pi t \in K \backslash 0$. Then $\phi-\mathfrak{T} \phi \neq \tau \sin \pi t$, for every $\phi \in \partial K_{R}$ and $\tau \geq 0$. If there exist $\phi_{0} \in \partial K_{R}$ and $\tau \geq 0$ such that $\phi_{0}-\mathfrak{T} \phi_{0}=\tau \sin \pi t$, then

$$
\phi_{0}^{(4)}(t)-b \phi_{0}^{\prime \prime}(t)-\tau\left(\pi^{4}+b \pi^{2}\right) \sin \pi t=-\phi_{0}(t) \phi_{0}^{\prime \prime}(t)+\mu f\left(t, \phi_{0}(t),-\frac{\phi_{0}^{\prime \prime}(t)}{\mu}\right)
$$

and

$$
\phi_{0}(0)=\phi_{0}(1)=\phi_{0}^{\prime \prime}(0)=\phi_{0}^{\prime \prime}(1)=0 \text {. }
$$

From $\left(F_{2}\right)$, it follows that

$$
\begin{aligned}
\phi_{0}^{(4)}(t)-b \phi_{0}^{\prime \prime}(t)= & -\phi_{0}(t) \phi_{0}^{\prime \prime}(t)+\mu f\left(t, \phi_{0}(t),-\frac{\phi_{0}^{\prime \prime}(t)}{\mu}\right) \\
& +\tau\left(\pi^{4}+b \pi^{2}\right) \sin \pi t \\
\geq & -\phi_{0}(t) \phi_{0}^{\prime \prime}(t)+\mu\left[\alpha_{2} \phi_{0}(t)-\beta_{2} \frac{\phi_{0}^{\prime \prime}(t)}{\mu}-h(t)\right] \\
\geq & \mu \alpha_{2} \phi_{0}(t)-\beta_{2} \phi_{0}^{\prime \prime}(t)-\mu h(t) .
\end{aligned}
$$

Multiplying this inequality by $\sin \pi t$ and integrating on $[0,1]$, we have

$$
\pi^{4} \int_{0}^{1} \phi_{0}(t) \sin \pi t d t \geq\left[\mu \alpha_{2}+\left(\beta_{2}-b\right) \pi^{2}\right] \int_{0}^{1} \phi_{0}(t) \sin \pi t d t-\mu \int_{0}^{1} h(t) \sin \pi t d t,
$$


which yields

$$
\int_{0}^{1} \phi_{0}(t) \sin \pi t d t \leq \frac{\mu \int_{0}^{1} h(t) \sin \pi t d t}{\mu \alpha_{2}+\left(\beta_{2}-b\right) \pi^{2}-\pi^{4}}
$$

Furthermore, we have

$$
\begin{aligned}
\left\|\phi_{0}\right\| & \leq\left(\Gamma+\frac{\pi^{3}}{4}\right) \int_{0}^{1} \phi_{0}(t) \sin \pi t d t \\
& \leq\left(\Gamma+\frac{\pi^{3}}{4}\right) \frac{\mu}{\frac{1}{2} \alpha_{2}+\left(\beta_{2}-b\right) \pi^{2}-\pi^{4}} \int_{0}^{1} h(t) \sin \pi t d t=\bar{R} .
\end{aligned}
$$

Let $R>\max \left\{\bar{R}, r_{1}, \mu r_{1}\right\}$. Then $i\left(\mathfrak{T}, K_{R}, K\right)=0$.

Finally,

$$
i\left(\mathfrak{T}, K_{R} \backslash K_{r}, K\right)=i\left(\mathfrak{T}, K_{R}, K\right)-i\left(\mathfrak{T}, K_{r}, K\right)=-1 .
$$

Therefore $\mathfrak{T}$ has a fixed point in $K_{R} \backslash K_{r}$, which is the positive solution of (1.5).

Corollary 3.2. Assume that $\left(F_{2}\right)$ holds. In addition, $f$ satisfies the following assumption:

$\left(F_{3}\right)$ There exist $\alpha_{3}>0, \beta_{3} \geq 0, r_{2}>0,0<\frac{\mu \alpha_{3}}{\pi^{4}}+\frac{\beta_{3}+\frac{\min \left\{1, \mu^{2}\right\}}{2} r_{2}-b}{\pi^{2}}<1$ such that

$$
f(t, \phi, u) \leq \alpha_{3} \phi+\beta_{3} u, \forall t \in I, \phi \in\left[0, r_{2}\right], u \in\left[0, \mu r_{2}\right] .
$$

Then (1.5) has at least one positive solution.

Proof. The proof is similar to Theorem 3.1. We only need to revise (3.1) as

$$
\begin{aligned}
\bar{\phi}^{(4)}(t)-b \bar{\phi}^{\prime \prime}(t) & =-\bar{\lambda} \bar{\phi}(t) \bar{\phi}^{\prime \prime}(t)+\bar{\lambda} \mu f\left(t, \bar{\phi}(t),-\frac{1}{\mu} \bar{\phi}^{\prime \prime}(t)\right) \\
& \leq-\bar{\lambda} \bar{\phi}(t) \bar{\phi}^{\prime \prime}(t)+\bar{\lambda} \mu\left[\alpha_{3} \bar{\phi}(t)-\beta_{3} \frac{1}{\mu} \bar{\phi}^{\prime \prime}(t)\right] \\
& \leq-\bar{\phi}(t) \bar{\phi}^{\prime \prime}(t)+\mu \alpha_{3} \bar{\phi}(t)-\beta_{3} \bar{\phi}^{\prime \prime}(t) \\
& \leq-\bar{\phi}^{\prime \prime}(t) \cdot \frac{\min \left\{1, \mu^{2}\right\}}{2} r_{2}+\mu \alpha_{3} \bar{\phi}(t)-\beta_{3} \bar{\phi}^{\prime \prime}(t) .
\end{aligned}
$$

Corollary 3.3. Assume that $\left(F_{2}\right)$ holds. In addition, $f$ satisfies the following assumption:

$\left(F_{4}\right)$ There exist $\alpha_{4}>0, \beta_{4} \geq 0, r_{3}>0,0<\frac{\mu \alpha_{4}+\left(\frac{\min \left\{1, \mu^{2}\right\}}{\pi^{4}} r_{3}\right)^{2}}{\pi^{4}}+\frac{\beta_{4}-b}{\pi^{2}}<1$ such that

$$
f(t, \phi, u) \leq \alpha_{4} \phi+\beta_{4} u, \forall t \in I, \phi \in\left[0, r_{3}\right], u \in\left[0, \mu r_{3}\right] .
$$

Then (1.5) has at least one positive solution.

Proof. The proof is similar to Theorem 3.1. We only need to revise (3.1) as

$$
\begin{aligned}
\bar{\phi}^{(4)}(t)-b \bar{\phi}^{\prime \prime}(t) & =-\bar{\lambda} \bar{\phi}(t) \bar{\phi}^{\prime \prime}(t)+\bar{\lambda} \mu f\left(t, \bar{\phi}(t),-\frac{1}{\mu} \bar{\phi}^{\prime \prime}(t)\right) \\
& \leq-\bar{\lambda} \bar{\phi}(t) \bar{\phi}^{\prime \prime}(t)+\bar{\lambda} \mu\left[\alpha_{4} \bar{\phi}(t)-\beta_{4} \frac{1}{\mu} \bar{\phi}^{\prime \prime}(t)\right] \\
& \leq-\bar{\phi}(t) \bar{\phi}^{\prime \prime}(t)+\mu \alpha_{4} \bar{\phi}(t)-\beta_{4} \bar{\phi}^{\prime \prime}(t) \\
& \leq\left(\frac{\min \left\{1, \mu^{2}\right\}}{2} r_{3}\right)^{2}+\mu \alpha_{4} \bar{\phi}(t)-\beta_{4} \bar{\phi}^{\prime \prime}(t)
\end{aligned}
$$


Corollary 3.4. Assume that $\left(F_{2}\right)$ holds. In addition, $f$ satisfies the following assumption:

$$
0<\frac{1}{2 \pi^{4}}+\frac{1-2 b}{2 \pi^{2}}+\mu \frac{\max _{(t, \phi, u) \in[0,1] \times[0,1] \times\left[0, \frac{1}{\mu}\right]} f(t, \phi, u)}{\pi^{4}}<1 .
$$

Then (1.5) has at least one positive solution.

Proof. The proof is similar to Theorem 3.1. Choosing $r<1$, we only need to revise (3.1) as

$$
\begin{aligned}
\bar{\phi}^{(4)}(t)-b \bar{\phi}^{\prime \prime}(t) & =-\bar{\lambda} \bar{\phi}(t) \bar{\phi}^{\prime \prime}(t)+\bar{\lambda} \mu f\left(t, \bar{\phi}(t),-\frac{1}{\mu} \bar{\phi}^{\prime \prime}(t)\right) \\
& \leq-\bar{\phi}(t) \bar{\phi}^{\prime \prime}(t)+\mu \max _{\left(t, \phi,-\phi^{\prime \prime}\right) \in[0,1] \times[0,1] \times[0,1]} f\left(t, \bar{\phi},-\frac{1}{\mu} \bar{\phi}^{\prime \prime}\right) \\
& \leq \frac{\phi(t)}{2}+\frac{-\bar{\phi}^{\prime \prime}(t)}{2}+\mu \max _{\left(t, \phi,-\phi^{\prime \prime}\right) \in[0,1] \times[0,1] \times[0,1]} f\left(t, \bar{\phi},-\frac{1}{\mu} \bar{\phi}^{\prime \prime}\right) .
\end{aligned}
$$

Theorem 3.5. Assume that $\left(F_{2}\right)$ holds. In addition, $f$ satisfies the following assumptions:

$\left(F_{4}\right)$ There exist $\tilde{R}>r_{4}>0, \alpha_{5}>0, \beta_{5} \geq 0, \frac{\mu \alpha_{5}}{\pi^{4}}+\frac{\beta_{5}-b}{\pi^{2}}>1$ such that

$$
\frac{7}{6}\left(\frac{\operatorname{coth} \sqrt{b}}{2 \sqrt{b}}-\frac{1}{2 b}\right)\left[\tilde{R}^{2}+\mu \max _{t \in[0,1] \times[0, \tilde{R}] \times\left[0, \frac{1}{\mu} \tilde{R}\right]} f(t, \phi, u)\right]<\tilde{R}
$$

and

$$
f(t, \phi, u) \geq \alpha_{5} \phi+\beta_{5} u, \forall t \in[0,1], \phi \in\left[0, r_{4}\right], u \in\left[0, \mu r_{4}\right] .
$$

Then (1.5) has at least two positive solutions.

Proof. Choosing $r=\frac{\min \left\{1, \mu^{2}\right\}}{2} r_{4}$ and $e=\sin \pi t \in K \backslash 0$, we have $\phi-\mathfrak{T} \phi \neq \tau \sin \pi t$, for every $\phi \in \partial K_{r}$ and $\tau \geq 0$. If there exist $\phi_{0} \in \partial K_{r}$ and $\tau \geq 0$ such that $\phi_{0}-\mathfrak{T} \phi_{0}=\tau \sin \pi t$, then

$$
\phi_{0}^{(4)}(t)-b \phi_{0}^{\prime \prime}(t)-\tau\left(\pi^{4}+b \pi^{2}\right) \sin \pi t=-\phi_{0}(t) \phi_{0}^{\prime \prime}(t)+\mu f\left(t, \phi_{0}(t),-\frac{\phi_{0}^{\prime \prime}(t)}{\mu}\right)
$$

and

$$
\phi_{0}(0)=\phi_{0}(1)=\phi_{0}^{\prime \prime}(0)=\phi_{0}^{\prime \prime}(1)=0 \text {. }
$$

For $\phi_{0} \in \partial K_{r}$, we have

$$
\left|\phi_{0}\right|_{\infty} \leq \frac{r_{4}}{2}, \quad\left|\frac{1}{\mu} \phi_{0}^{\prime \prime}\right|_{\infty} \leq \frac{\frac{\min \left\{1, \mu^{2}\right\}}{2}}{\mu} r_{4} \leq \frac{\frac{\mu^{2}}{2}}{\mu} r_{4}<\mu r_{4} .
$$

From $\left(F_{4}\right)$, it follows that

$$
\begin{aligned}
\phi_{0}^{(4)}(t)-b \phi_{0}^{\prime \prime}(t)= & -\phi_{0}(t) \phi_{0}^{\prime \prime}(t)+\mu f\left(t, \phi_{0}(t),-\frac{\phi_{0}^{\prime \prime}(t)}{\mu}\right) \\
& +\tau\left(\pi^{4}+b \pi^{2}\right) \sin \pi t \\
\geq & -\phi_{0}(t) \phi_{0}^{\prime \prime}(t)+\mu\left[\alpha_{5} \phi_{0}(t)-\beta_{5} \frac{\phi_{0}^{\prime \prime}(t)}{\mu}\right] \\
\geq & \mu \alpha_{5} \phi_{0}(t)-\beta_{5} \phi_{0}^{\prime \prime}(t) .
\end{aligned}
$$

Multiplying this inequality by $\sin \pi t$ and integrating on $[0,1]$, we have

$$
\pi^{4} \int_{0}^{1} \phi_{0}(t) \sin \pi t d t \geq\left[\mu \alpha_{5}+\left(\beta_{5}-b\right) \pi^{2}\right] \int_{0}^{1} \phi_{0}(t) \sin \pi t d t .
$$


Since $\int_{0}^{1} \phi_{0}(t) \sin \pi t d t>0$, we have

$$
\pi^{4} \geq \mu \alpha_{5}+\left(\beta_{5}-b\right) \pi^{2}
$$

which contradicts $\left(F_{4}\right)$. Then, $i\left(\mathfrak{T}, K_{r}, K\right)=0$.

Now, we prove that $\lambda \mathfrak{T} \phi \neq \phi$ for $\phi \in \partial K_{\tilde{R}}$ and $0<\lambda \leq 1$. For any $\phi \in \partial K_{\tilde{R}}$, we have

$$
\begin{aligned}
\|\lambda \mathfrak{T} \phi\| \leq & \|\mathfrak{T} \phi\|=|\mathfrak{T} \phi|_{\infty}+\left|(\mathfrak{T} \phi)^{\prime \prime}\right|_{\infty} \\
\leq & \left|\int_{0}^{1} \int_{0}^{1} G(t, s) G_{b}(s, \tau) \phi(\tau) \phi^{\prime \prime}(\tau) d \tau d s\right|_{\infty} \\
& +\left|\mu \int_{0}^{1} \int_{0}^{1} G(t, s) G_{b}(s, \tau) f\left(\tau, \phi(\tau), \frac{-\phi^{\prime \prime}(\tau)}{\mu}\right) d \tau d s\right|_{\infty} \\
& +\left|\int_{0}^{1} G_{b}(t, \tau) \phi(\tau) \phi^{\prime \prime}(\tau) d \tau\right|_{\infty} \\
& +\left|\mu \int_{0}^{1} G_{b}(t, \tau) f\left(\tau, \phi(\tau), \frac{-\phi^{\prime \prime}(\tau)}{\mu}\right) d \tau\right|_{\infty} \\
\leq & \frac{1}{6}\left(\frac{\operatorname{coth} \sqrt{b}}{2 \sqrt{b}}-\frac{1}{2 b}\right) \tilde{R}^{2}+\mu \frac{1}{6}\left(\frac{\operatorname{coth} \sqrt{b}}{2 \sqrt{b}}-\frac{1}{2 b}\right)_{t \in[0,1] \times[0, \tilde{R}] \times\left[0, \frac{1}{\mu} \tilde{R}\right]} f\left(t, \phi,-\frac{1}{\mu} \phi^{\prime \prime}\right) \\
& +\left(\frac{\operatorname{coth} \sqrt{b}}{2 \sqrt{b}}-\frac{1}{2 b}\right) \tilde{R}^{2}+\mu\left(\frac{\operatorname{coth} \sqrt{b}}{2 \sqrt{b}}-\frac{1}{2 b}\right)_{t \in[0,1] \times[0, \tilde{R}] \times\left[0, \frac{1}{\mu} \tilde{R}\right]} f\left(t, \phi,-\frac{1}{\mu} \phi^{\prime \prime}\right) \\
= & \frac{7}{6}\left(\frac{\operatorname{coth} \sqrt{b}}{2 \sqrt{b}}-\frac{1}{2 b}\right) \tilde{R}^{2}+\mu \frac{7}{6}\left(\frac{\operatorname{coth} \sqrt{b}}{2 \sqrt{b}}-\frac{1}{2 b}\right)_{t \in[0,1] \times[0, \tilde{R}] \times\left[0, \frac{1}{\mu} \tilde{R}\right]} f\left(t, \phi,-\frac{1}{\mu} \phi^{\prime \prime}\right) \\
< & \tilde{R},
\end{aligned}
$$

which implies that $i\left(\mathfrak{T}, K_{\tilde{R}}, K\right)=1$. In the same proof of Theorem 3.1, it follows from $\left(F_{2}\right)$ that there exist a sufficiently large

$$
R>\max \left\{\frac{\min \left\{1, \mu^{2}\right\}}{2} r_{4}, \tilde{R}, \bar{R}\right\}
$$

such that $i\left(\mathfrak{T}, K_{R}, K\right)=0$.

Finally,

$$
\begin{gathered}
i\left(\mathfrak{T}, K_{\tilde{R}} \backslash K_{r}, K\right)=i\left(\mathfrak{T}, K_{\tilde{R}}, K\right)-i\left(\mathfrak{T}, K_{r}, K\right)=1, \\
i\left(\mathfrak{T}, K_{R} \backslash K_{\tilde{R}}, K\right)=i\left(\mathfrak{T}, K_{R}, K\right)-i\left(\mathfrak{T}, K_{\tilde{R}}, K\right)=-1,
\end{gathered}
$$

Therefore $\mathfrak{T}$ has two fixed points in $K_{\tilde{R}} \backslash K_{r}$ and $K_{R} \backslash K_{\tilde{R}}$, which are the positive solutions of (1.5).

Example 3.6. Let us consider the following system

$$
\left\{\begin{array}{l}
-u^{\prime \prime}+u=\phi u+\frac{1}{10}\left\{2 \pi^{4}\left(e^{\phi}-1\right)+u^{2}+2 u\right\}, 0<t<1 \\
-\phi^{\prime \prime}=\frac{1}{10} u \\
\phi(0)=\phi(1)=0, \phi^{\prime \prime}(0)=\phi^{\prime \prime}(1)=0
\end{array}\right.
$$

Let

$$
\alpha_{1}=2 \pi^{4} e, \beta_{1}=3, r_{1}=1
$$


It is clear that

$$
\begin{aligned}
0 & <\frac{\mu \alpha_{1}}{\pi^{4}}+\frac{\beta_{1}-b}{\pi^{2}}+\frac{\min \left\{1, \mu^{2}\right\}}{2 \pi^{4}} r_{1} \\
& =\frac{2 e}{10}+\frac{2}{\pi^{2}}+\frac{1}{200 \pi^{4}} \\
& <1 .
\end{aligned}
$$

For $t \in[0,1], \phi \in[0,1], u \in\left[0, \frac{1}{10}\right]$, we have

$$
f(t, \phi, u)=2 \pi^{4}\left(e^{\phi}-1\right)+u^{2}+2 u \leq 2 \pi^{4} e \phi+u+2 u \leq \alpha_{1} \phi+\beta_{1} u,
$$

which yields that $\left(F_{1}\right)$ holds.

On the other hand, let

$$
\alpha_{2}=2 \pi^{4}, \beta_{2}=2, h(t)=t^{2}
$$

Then,

and

$$
\frac{\alpha_{2}}{2 \pi^{4}}+\frac{\beta_{2}-b}{\pi^{2}}=1+\frac{1}{\pi^{2}}>1
$$

$$
f(t, \phi, u)=2 \pi^{4}\left(e^{\phi}-1\right)+u^{2}+2 u \geq 2 \pi^{4} \phi+2 u \geq \alpha_{2} \phi+\beta_{2} u-h(t),
$$

which guarantee that $\left(F_{2}\right)$ holds. Therefore, by Theorem 3.1, (3.3) has at least one positive solution.

Example 3.7. Let us consider the following system

$$
\left\{\begin{array}{l}
-u^{\prime \prime}+u=\phi u+\frac{\phi}{2+\sin u}+\left(\pi^{2}+1\right) e^{u}+\cos u-\sin t, 0<t<1 \\
-\phi^{\prime \prime}=\frac{1}{156} u \\
\phi(0)=\phi(1)=0, \phi^{\prime \prime}(0)=\phi^{\prime \prime}(1)=0
\end{array}\right.
$$

Let

It is clear that

$$
\alpha_{2}=\frac{1}{3}, \beta_{2}=\pi^{2}+1
$$

and

$$
\frac{\alpha_{2}}{2 \pi^{4}}+\frac{\beta_{2}-b}{\pi^{2}}=\frac{1}{2 \pi^{4}}+1>1
$$

$$
f(t, \phi, u)=\frac{\phi}{2+\sin u}+\left(\pi^{2}+1\right) e^{u}+\cos u-\sin t \geq \alpha_{2} \phi+\beta_{2} u-(\sin t+1),
$$

which yields that $\left(F_{2}\right)$ holds.

On the other hand, let

$$
\alpha_{5}=\frac{1}{3}, \beta_{5}=\pi^{2}+1, \tilde{R}=1, r_{4}=\frac{1}{2}
$$

Then,

In addition, we also have

$$
\frac{\mu \alpha_{5}}{\pi^{4}}+\frac{\beta_{5}-b}{\pi^{2}}>1
$$

$$
\begin{aligned}
& \frac{7}{6}\left(\frac{\operatorname{coth} \sqrt{b}}{2 \sqrt{b}}-\frac{1}{2 b}\right)\left[\tilde{R}^{2}+\mu \max _{t \in[0,1] \times[0, \tilde{R}] \times\left[0, \frac{1}{\mu} \tilde{R}\right]} f(t, \phi, u)\right] \\
& \leq \frac{7}{6\left(e^{2}-1\right)}\left[1+\frac{\frac{1}{2}+\left(\pi^{2}+1\right) e^{\frac{1}{156}}+1}{156}\right]<1=\tilde{R}
\end{aligned}
$$


and

$$
\begin{aligned}
f(t, \phi, u) & =\frac{\phi}{2+\sin u}+\left(\pi^{2}+1\right) e^{u}+\cos u-\sin t \\
& \geq \alpha_{5} \phi+\beta_{5} u
\end{aligned}
$$

for $t \in[0,1], \phi \in\left[0, \frac{1}{2}\right], u \in\left[0, \frac{1}{312}\right]$, so that $\left(F_{4}\right)$ holds. Therefore, by Theorem 3.5, (3.4) has at least two positive solution.

\section{Funding}

The authors were supported by the Fundamental Research Funds for the Central Universities (2018B58614).

\section{REFERENCES}

[1] R. Benguria, H. Brezis, E.H. Lieb, The Thomas-Fermi-Von Weizsäker theory of atoms and molecules, Comm. Math. Phys. 79 (1981), 167-80.

[2] E.H. Lieb, Thomas-Fermi and related theories and molecules, Rev. Modern Phys. 53 (1981), 603-641.

[3] P. Markowich, C. Ringhofer, C. Schmeiser, Semiconductor Equations, Springer-Verlag, New York, 1990.

[4] W.E. Kastenberg, P.L. Chambré, On the stability of nonlinear space-dependent reactor kinetics, Nucl. Sci. Eng. 31 (1968) 67-79.

[5] Y. Gu, M. Wang, Existence of positive stationary solutions and threshold results for reaction-diffusion system, J. Differential Equations 130 (1996), 277-291.

[6] F. Wang, Y. An, Positive solutions for a second-order differential system, J. Math. Anal. Appl. 373 (2011), 370-375.

[7] R. Chen, R. Ma, Positive solutions of the second-order differential systems in reactor dynamics, Appl. Math. Comput. 219 (2012), 3882-3892.

[8] F. Wang, Y. An, On positive solutions for a second order differential system with indefinite weight, Appl. Math. Comput. 259 (2015), 753-761.

[9] A. Cabada, S. Tersian, Multiplicity of solutions of a two point boundary value problem for a fourth-order equation, Appl. Math. Comput. 219 (2013), 5261-5267.

[10] Q. Dang, T. Ngo, Existence results and iterative method for solving the cantilever beam equation with fully nonlinear term, Nonlinear Anal. Real World Appl. 36 (2017), 56-68.

[11] J.M. do Ó, S. Lorca, P. Ubilla, Local superlinearity for elliptic systems involving parameters, J. Differential Equations 211 (2005), 1-19.

[12] J.M. do Ó, S. Lorca, J. Sanchez, P. Ubilla, Positive solutions for a class of multiparameter ordinary elliptic systems, J. Math. Anal. Appl. 332 (2007) 1249-1266.

[13] J. R. Graef, L. Kong, A necessary and sufficient condition for existence of positive solutions of nonlinear boundary value problems, Nonlinear Anal. 66 (2007), 2389-2412.

[14] Y. Lee, Multiplicity of positive radial solutions for multiparameter semilinear elliptic systems on an annulus, J. Differential Equations 174 (2001), 420-441.

[15] Y. Li, Positive solutions of fourth-order boundary value problems with two parameters, J. Math. Anal. Appl. 281 (2003), 477-484.

[16] Y. Li, Multiply sign-changing solutions for fourth-order nonlinear boundary value problems, Nonlinear Anal. 67 (2007), 601-608.

[17] Y. Li, Existence of positive solutions for the cantilever beam equations with fully nonlinear terms, Nonlinear Anal. Real World Appl. 27 (2016), 221-237.

[18] Y. Li, On the existence of positive solutions for the bending elastic beam equations, Appl. Math. Comput. 189 (2007), 821-827.

[19] R. Ma Nodal solutions of boundary value problems of fourth-order ordinary differential equations, J. Math. Anal. Appl. 319 (2006), 424-434.

[20] T. F. Ma, Existence results for a model of nonlinear beam on elastic bearings, Appl. Math. Lett. 13 (2000), 11-15. 
[21] T. F. Ma, Existence results and numerical solutions for a beam equation with nonlinear boundary conditions, Appl. Numer. Math. 47 (2003), 189-196.

[22] T. F. Ma, Positive solutions for a nonlocal fourth order equations of Kirchhoff type, Discrete Contin. Dyn. Syst. 2007 (2007), 694-703.

[23] D. O'Regan, Solvability of some fouth (and higher) order singular boundary problems, J. Math. Anal. Appl. 161 (1991), 78-116.

[24] Y. Wei, Q. Song, Z. Bai, Existence and iterative method for some fourth order nonlinear boundary value problems, Appl. Math. Lett. 87 (2019), 101-107.

[25] D. Guo, V. Lakshmikantham, Nonlinear Problems in Abstract Cones, Academic Press, New York, 1988. 